\title{
RESPONSABILIDAD SOCIAL EMPRESARIAL DE LAS ALIANZAS PÚBLICO PRIVADAS EN PUERTOS HONDUREÑOS, UN ANÁLISIS FINANCIERO

\author{
Cintia Liliana Salgado Gómez*
}

DOI: I0.5377/EYA.VI2II.I 2957

Recibido: 06/06/2020 Aceptado: 29/11/2021

\section{RESUMEN}

La Responsabilidad Social Empresarial (RSE) debe desarrollar una relación simbiótica entre el éxito de la empresa, como el de la comunidad (Porter \& Kramer, 2006). Honduras adopta, en año 2012, el modelo APP para puertos hondureños, por lo que realizar una valoración de este se vuelve indispensable, en el sentido de que hay una obvia necesidad de asegurar que la alianza ha sido de beneficio para las partes. Se realizó un análisis financiero de la RSE de las APPs y de la APP en específico. Lo importante de este análisis de tipo empírico, es la evaluación de la información, generada producto del seguimiento de los proyectos por parte de los entes fiscalizadores para este efecto, misma que sin duda desvela que con pocos años de operación, la rentabilidad del socio privado es atractiva. A su vez refleja que, al examinar el proceso, la metodología financiera y los datos utilizados para la evaluación del modelo previo a su implementación, se basaron en la transferencia de riesgo y la relación calidad-productividad. Los modelos de APP en Honduras están en proceso de madurez, por lo que la información generada a la fecha en seguimiento a lo establecido en el contrato debe seguir un proceso más riguroso.

PALABRAS CLAVES: responsabilidad social, empresas públicas, público-privadas, adquisición.

JEL: M14, L32, H57

*Doctoranda del Doctorado en Dirección Empresarial de la Facultad de Ciencias Económicas, Administrativas y Contables, Universidad Nacional Autónoma de Honduras. https://orcid.org/0000-0001-7137-0816 Correo electrónico: cintia.1.salgado@gmail.com Tegucigalpa, Honduras. 


\title{
Corporate Social Responsibility of Public-Private Partnerships in Honduran Ports, A Financial Analysis
}

Cintia Liliana Salgado Gómez*

DOI: I0.5377/EYA.VI2II.I2957

Received: 06/06/2020 Accepted: 29/11/2021

\begin{abstract}
Corporate Social Responsibility must develop a symbiotic relationship between the success of the company and the community (Porter \& Kramer, 2006). Honduras, in 2012, adopts the PPP model for Honduran ports, so making an assessment of it becomes essential, in the sense that there is an obvious need to ensure that the alliance has been of benefit to the parties. A financial analysis of the CSR of the APPs and the APP itself was carried out. The important thing about this empirical analysis is the evaluation of the information generated, product of the monitoring of the projects by the supervisory entities for this purpose, which undoubtedly reveals that, with a few years of operation, the profitability of the Private partner is attractive, in turn it reflects that, when examining the process, the financial methodology and the data used for the evaluation of the model prior to its implementation, they were based on risk transfer and the quality-productivity ratio. The PPP models in Honduras are in the process of maturity, therefore, the information generated to date, in compliance with the provisions of the contract, must follow a more rigorous process.
\end{abstract}

KEYWORDS: social responsibility, public enterprises, public-private enterprises, procurement.

JEL: M14, L32, H57

*Doctoral candidate for the Doctorate in Business Management at the Faculty of Economic, Administrative and Accounting Sciences, National Autonomous University of Honduras. https://orcid.org/0000-0001-7137-0816 Email: cintia.1.salgado@gmail.com Tegucigalpa, Honduras. 


\section{INTRODUCCIÓN}

En el contexto mundial, caracterizado por una desaceleración de la producción y del comercio, los países de América Latina y el Caribe deben redoblar esfuerzos para promover el crecimiento económico, incrementar la productividad y el desarrollo social de la región.

Según la CEPAL, (2016), la articulación de esfuerzos hacia un cambio estructural con igualdad y sostenibilidad ambiental necesita una nueva ecuación entre el Estado, el mercado y la sociedad civil, en la que los actores públicos y privados colaboren por medio de asociaciones novedosas que aborden el desarrollo social y sustentable a través de inversiones conjuntas. El apoyo incondicional por parte de la empresa privada y la sociedad en general, a través de una Responsabilidad Social acertada, es clave para crear la sinergia de éxito en la Sociedad. Por ello, siguiendo las diferentes dinámicas de negocio, como por ejemplo las Alianzas Público-Privadas que en los últimos años han sido un mecanismo o herramienta con las que los gobiernos de América Latina a partir de la década de los noventa han buscado mejorar los servicios que ofrecen a través de infraestructura existente o nueva de la mano con el sector privado.

Por lo anterior, se vuelve de vital importancia analizar si los servicios que se prestan a la ciudadanía a través de este tipo de modelo de negocio están cumpliendo con los requerimientos solicitados por cada gobierno, así como evaluar si la proyección a través de una Responsabilidad Social Empresarial estratégica está contribuyendo al desarrollo social de la comunidad.

El presente documento contiene un estudio exploratorio sobre la valoración financiera de la Responsabilidad Social Empresarial de las Alianzas Público Privadas aplicado en Honduras para los convenios firmados en el sector portuario.

El análisis que se presenta en este estudio, es con base a información proporcionada por los entes competentes para el seguimiento de las APP en Honduras. 


\section{Marco teórico}

La Responsabilidad Social Empresarial (RSE), así como las Alianzas Público Privadas (APP) datan de tiempos antiguos y, estas últimas tienen como fin proveer bienes y servicios de la óptima calidad para mejorar la condición de vida de los ciudadanos.

La acción de la Responsabilidad Social Empresarial tiene sus orígenes antes de Cristo (Herrera, J.C. \& Abreu Jose, 2008) y la filosofía del Zaratrusta tiene gran relevancia por sus tres pilares fundamentales: a) buenos pensamientos, b) buenas palabras y c) buenas acciones. Correa, Juan G. (2007) distingue tres fases que posibilitan una mejor comprensión del concepto RSE, (a) la fase inicial: no existe un marco conceptual, sin embargo, algunos empresarios desarrollan actividades de tipo filantrópica y asistencia social, (b) la fase primera, se desarrolla un criterio más social para el bienestar común de las empresas y el entorno que las rodean, (c) fase segunda, las organizaciones asumen un rol más crítico, con respecto a su accionar empresarial y los efectos que esta podía tener sobre el entorno que los rodean, el Estado inicia un proceso de vigilancia y control para salvaguardar los intereses públicos y el medio ambiente. (d) fase tercera, se caracteriza por una interrelación más estrecha entre Estado y empresa, existen políticas reguladoras, permitiendo así un compromiso serio y coherente y de largo plazo ante las necesidades de la sociedad.

Sin embargo, Araque Padilla \& Montero Simo (2008) son de la opinión de que el concepto de RSE ha pasado por un proceso de evolución de diferentes momentos: (a) un paternalismo empresarial, (b) acciones sociales en respuesta al temor a nuevas regulaciones, (c) equilibrio de intereses diversos a través de la nueva figura del directivo, (d) desarrollo conceptual, (e) expansión de la investigación práctica, (f) nuevos temas de interés: business ethics o teoría de los stakeholders, entre otros.

Por lo anterior, se puede observar que pese a los procesos evolutivos del concepto de RSE, el mismo "es variado, y como tal genera polémicas y es fuente de discusión" (Sarmiento del Valle, 2011, Pag. 7). Por un lado, la Comisión de las Comunidades Europeas (2001) indica que "la responsabilidad social de las empresas es, esencialmente, un concepto con arreglo al cual las empresas deciden voluntariamente contribuir al logro de una sociedad mejor y un medio ambiente más limpio". (Pag. 4). Opinan Ruiz Malbarez \& Romero González (2011), que la RES es "visto como un proceso de actuación empresarial donde intervienen sujetos activos y pasivos, internos y externos, matizados por factores objetivos y subjetivos"(Pag. 127). Indica nuevamente Sarmiento del Valle, (2011), que la RES es una continua relación con la sociedad a todos los niveles. "que involucra a todos sus stakeholders y debe formar parte de la planeación estratégica de las empresas que la desarrollan” (Pag. 7). Al igual Bestratén y Pujol (2004), dan su opinión citando que la RSE se presenta cuando la empresa ofrece (a) productos y servicios que responden a las necesidades de sus usuarios, de tal manera que contribuyan a su bienestar; (b) tiene un comportamiento que va más allá del cumplimiento de los estándares mínimos reglamentarios; (c) demuestra conducta ética en todas sus acciones; (d) brinda condiciones saludables y seguras a sus trabajadores; (e) muestra respeto estricto por el ambiente, interno y externo y, está integrada a la comunidad, (e) conoce y participa de sus anhelos y necesidades, así como de sus problemas. Para Porter \& Kramer (2006) "se debe medir y hacer público el desempeño social, siempre y cuando los indicadores se midan en forma consistente y reflejen apropiadamente el impacto social”. (Pag.5).

Por lo tanto, para efectos de esta investigacion la Responsabilidad Social Empresarial se definirá como: Un valor compartido al invertir en aspectos sociales de contexto que fortalecen la competitividad de la empresa. Y donde se desarrolla una relación simbiótica: el éxito de la empresa y el de la comunidad se refuerzan mutuamente. Cuanto más estrechamente vinculado está un tema social con el negocio de la empresa, mayor es la oportunidad de aprovechar 
los recursos y capacidades de esta para beneficiar a la sociedad, asi como medir de forma consistente y apropiada el desempeño social. (Porter \& Kramer, 2006, Pags. 5 y 12).

Por su lado, las Alianzas Público Privadas (APP) también remontan sus orígenes a tiempos antiguos. Según Wettenhall (2003); Ascheri (2003-2006); Hodge \& Greve (2007), se pueden citar algunos ejemplos como: (a) Mateo de la Biblia con su trabajo como recolector de impuestos previo a convertirse en discípulo de Jesucristo, (b) la aparición de las ciudades-estado italianas, previo al siglo XV, así como la época dorada holandesa del siglo XVIII donde la habilidad de conjugar intereses públicos y privados dio como resultado la prosperidad económica, (c) el 82\% de los 197 buques de la armada de Sir Francis Drake que conquistaron la armada española en 1588, eran contratistas privados del almirante. Por lo anterior, la misma historia revela una y otra vez que siempre ha existido cierto grado de cooperación entre el sector público y el privado (Wettenhall 2003: 2005).

Por lo tanto, indica Wettenhall (2005), que "no hay nada nuevo acerca de la combinación de los esfuerzos públicos y privados ... lo que los nuevos entusiastas puedan pensar.” (P. 34). Según Neto, Cruz, Rodrigues, \& Silva (2016), en un estudio bibliométrico, evidencian que la información sobre las APP sobrepasa los 600 artículos académicos, los cuales datan de los años 90.

Para Hodge (2004) existe eficiencia y la responsabilidad del riesgo por la parte privada involucrada. Al contrario, Nisar (2007) considera que los sectores que trabajan en asociación deben enfocarse en las áreas que cada uno mejor realiza. Sin embargo, Villalba-Romero \& Liyanage (2016) enfatizan el hecho de una relación estrecha entre las partes involucradas para que se logre un beneficio mutuo. Así mismo, Cui, Liu, Hope, \& Wang (2018) destacan el hecho de la existencia de una mejora en el valor económico de los productos de infraestructura del sector público.

El concepto APP abarca al menos cinco familias de acuerdos (Vaillancourt Rosenau, ed. 2000; Savas, 2000; Perrot \& Chatelus, eds. 2000; Osborne, 2001a: 2001b; Van Ham \& Koppenjan, 2001: 2002; Berg, Pollitt, \& Tsuji, 2002; Ghobadian, O’Regan, Gallear, \& Viney, 2004; Grimsey \& Lewis, 2004;Bovaird, 2004; Klijn \& Teisman, 2005; Hodge, 2007) que a su vez incluyen diferentes tipos de modelos contractuales: (a) cooperación institucional para la producción conjunta y el riesgo compartido (como la Autoridad Portuaria de los Países Bajos), (b) contratos de infraestructura a largo plazo (LTIC), (como se ejemplifica en los proyectos de la Iniciativa de Finanzas Privadas del Reino Unido), (c) redes de políticas públicas (en las cuales se enfatizan las relaciones entre partes interesadas), (d) sociedad civil y desarrollo comunitario, (e) renovación urbana y desarrollo económico en el centro de la ciudad (y en Estados Unidos se busca una cartera de medidas de desarrollo económico local y de re-crecimiento urbano).

Por consiguiente, se indica que las Alianzas Público Privadas son reconocidas como una convergencia de cooperación entre el sector público y privado (Villalba-Romero, 2016). Entre los cuales están los modelos de contratación APP, Private Finance Initiative (PFI) utilizados para entregar infraestructura pública, de servicio o ambos, por lo general en los sectores de transporte, energía, medio ambiente, la salud, la seguridad o la educación (Froud, 2003; Neto et al., 2016); Los de APP’s (McKee, Tiong, 1990; Edwards, \& Atunc, 2006; Alborta, Stevenson, Triana \& Sergio, 2011; Villalba-Romero, 2016; Cui et al., 2018); como ser Build Operate Transfer (BOT), Private-Public-Partnership (PPP), Private Finance Initiative (PFI), Build Own Operate (BOO), Build Own Operate Transfer (BOOT), Build Transfer Operate (BTO), Reconstruct Operate Transfer (ROT), Transfer Operate Transfer (TOT), Infrastructure, Privatized Infrastructure (PI), Privately Financed (PF), Design, Build, Finance and Operate (DBFO), Non-Profit Distribution (NPD), Franchising, Buy-Own- Lease Back (BOLB), Alzira model. Los autores citados previamente han determinado estos modelos conforme a las condiciones firmadas contractualmente, así como las variantes en los mismos. 


\subsection{Caracterización del SECTOR PORTUARIo de Honduras}

Para ver referencia de significados avocarse al Anexo 1. Honduras cuenta con seis puertos a nivel nacional, en la zona norte se encuentran cinco de ellos, Puerto Cortés, Tela, Puerto Castilla, La Ceiba, y Roatán; En la zona sur, cuenta con uno, San Lorenzo. De los seis puertos, Puerto Cortes es el más importante del país y maneja alrededor del 90\% del comercio marítimo. Por lo anterior, en el año 2013 se inició el proyecto de modernización de dos áreas importantes bajo la modalidad de APP, (a) la Terminal de Contenedores y Carga General de Puerto Cortés y (b) Terminal de Gráneles Sólidos de Puerto Cortés. Según información del portal web de la Comisión para la Promoción de la Alianza Público-Privada (COALIANZA), el modelo de APP utilizado para Puerto Cortés es Design Finance Build Operate Transfer (DFBOT). Sin embargo, no se encontró evidencia de estudios académicos debidamente publicados, a excepción de un documento presentado y llamado "Hybrid Annuity Projects: - Study of HAM in Highway Construction Projects-Owner and Contractor Prospective" donde indica que el DFBOT es un híbrido derivado del modelo BOT.

El modelo de contrato DFBOT se firmó con la Operadora Portuaria Centroamericana (OPC). La Operadora Portuaria Centroamericana es parte del grupo ICSTI con casa matriz en Filipinas. ICSTI está en el negocio de adquirir, desarrollar, manejar y operar terminales portuarias de contenedores en todo el mundo con una amplia experiencia en la materia.

\subsection{Perspectiva del ConcePto FinANCIERo}

Evaluar la efectividad de las APP con respecto al precio, la calidad en los contratos a largo plazo (LTIC) y la Responsabilidad Social Empresarial es un gran reto, ya que, si la alianza resulta ser un éxito, los análisis reflejarán si el Gobierno realmente no incurrió en costos durante el proceso, pero si la alianza fracasa, el riesgo será asumido en gran parte por el Gobierno. (Hodge, 2009). De acuerdo con Hodge (2010), no hay evidencia de metaanálisis o revisiones estadísticas sobre los LTIC. Sin embargo, indica Arthur Anderson and LSE Enterprise (2000), que del análisis realizado en 29 casos de negocios APP's en el Reino Unido, se reflejó en primer lugar que las estimaciones iniciales en referencia a las eficiencias que se obtendrían a través de LTIC incluyeron una cifra de ahorro de costos. Segundo, que las APP reducirían la presión sobre los presupuestos del sector público. Y tercero, la proporción de un mejor valor del dinero para los contribuyentes. De lo anterior, Hodge (2010) opina que únicamente el enunciado tercero es la promesa de política digna de un examen serio. Por otro lado, Edwards \& Shaoul (2003); NAO (2003); Shaoul (2005); Nisar (2007) y Marques \& Berg (2011) argumentan que el valor financiero se puede medir contra una serie de indicadores y los costos de evaluación comparativa donde la relación calidad-precio y la transferencia del riesgo es la principal justificación para elegir APP y es claro que transferir el riesgo a la parte privada aumentará el precio del proyecto. Por lo tanto, para el caso de Honduras, se analizará la información proveniente de: (a) montos de inversión, (b) ingresos para la parte privada y por Canon al Estado de Honduras y (c) mejoras en la productividad del puerto.

De acuerdo con Alborta et al. (2011), los modelos contractuales de Alianzas Público Privadas pueden segmentarse en (a) prestación de servicio. (b) Administración. (c) Concesiones. (d) BOT. (e) Cooperativas. (f) Asociaciones a riesgo compartido. Y dentro de estas clasificaciones pueden existir diferentes tipos de caracterización de modelos.

\section{Metodología}

El presente estudio está enmarcado en hacer una Valoración de la Responsabilidad Social Empresarial de las Alianzas Público Privadas en Puertos Hondureños desde un Análisis Financiero. 


\subsection{Objetivo General}

Valuar la Responsabilidad Social Empresarial de las Alianzas Público Privadas en Puertos Hondureños desde un Análisis Financiero.

\subsection{VARIABLES}

Las variables del estudio son dos, (a) La variable independiente: Responsabilidad Social Empresarial de las Alianzas Público Privadas en Puertos Hondureños. (b) La Variable dependiente: Análisis Financiero.

El análisis financiero dependerá del tipo de RSE de APP que se ha implementado en Puerto Cortés. Por lo tanto, se espera que la relación sea de tipo lineal simple, donde:

(a)El análisis Financiero es: F y, (b) La RSE de APP es: RSEAPP.

Por lo tanto, la relación debe ser:

$$
F=f(R s e A p p)
$$

donde RseApp = RseApp1, RseApp2, RseApp3...

\subsection{CARACTERIZACIÓN DE LA INVESTIGACIÓN}

La investigación es de tipo (a) Mixta, (b) Descriptiva y (c) Transversal.

\subsection{Caracterización del Estudio}

El estudio se caracteriza por ser una investigación de tipo: (a) cuantitativa, (b) empírica y (c) transversal.

\subsection{Población y Muestra del Estudio}

A continuación, se presenta en la tabla 1 con los datos de la población y muestra del estudio:

\section{Tabla 1 Población y Muestra en Estudio}

\begin{tabular}{|c|c|}
\hline $\begin{array}{l}\text { Población de Estudio (N) } \\
\mathrm{N}=2\end{array}$ & La RSE de Alianzas Público Privadas en los Puertos Hondureños. \\
\hline Criterios de Inclusión y Exclusión & $\begin{array}{l}\text { 1) Criterios de Inclusión: (a) Plan de RSE (b) Estudios académicos sobre } \\
\text { las Alianzas Público Privadas en otros países, (b) Información estadística y } \\
\text { financiera de los resultados de las APP en los puertos hondureños. } \\
\text { 2) Criterios de exclusión: Aquella información no relevante a los } \\
\text { resultados de las APP en la gestión de los puertos hondureños. }\end{array}$ \\
\hline Marco Muestral & La RSE de Alianzas Público Privadas en los Puertos Hondureños. \\
\hline $\begin{array}{l}\text { Muestra (n) } \\
n=2\end{array}$ & La RSE de Alianzas Público Privadas en Puerto Cortés, Honduras. \\
\hline Unidad de Análisis & $\begin{array}{l}\text { Valoración de la RSE de Alianzas Público Privadas en los puertos hondureños: un } \\
\text { análisis Financiero. }\end{array}$ \\
\hline
\end{tabular}

Fuente: Vindas, J (2019) Ficha de trabajo 2. Elaboración propia. 


\section{Desarrollo}

En la sección de desarrollo y análisis de información se estará realizando el análisis financiero del modelo APP en Puerto Cortes y el análisis financiero de las actividades RSE en la zona. Es importante aclarar que la información financiera con respecto a la RSE es escasa y se limita a la información publicada por el concesionario. En seguimiento a la información generada por la Operadora Portuaria Centroamericana de datos cualitativos sobre las actividades RSE realizadas en Puerto Cortes; La Superintendencia de Alianzas Público Privadas (SAPP) para Honduras, Empresa Nacional Portuaria (ENP) y COALIANZA donde, a través de los informes anuales, se presenta el seguimiento y resultados operativos de los proyectos en lo que respecta a los puertos hondureños. De la información cuantitativa generada en dichos informes y para efectos de este estudio se ha tomado en consideración la siguiente información: (a) inversión acumulada en millones de dólares al 31 de diciembre 2017. (b) Cantidad de contenedores transitados para importación como exportación. (c) Ingresos en millones de dólares para la parte privada, así como el canon del Estado de Honduras. (d) Inversión en RSE. (e) Reducción en la generación de desechos no reciclables.

Tal y como se muestra en la Tabla 2, actualmente existen en Honduras 11 proyectos con APP, cuya inversión al 31 de diciembre 2017 asciende a USD \$520.31 millones de dólares. De los cuales, el 29.76\% de la inversión se realizó en dos proyectos portuarios, la terminal de contenedores (USD\$95.41 millones) y la terminal de gráneles (USD\$59.40 millones), ambos ubicados en Puerto Cortés respectivamente.

Tabla 2: Alianzas Público Privadas, Honduras Inversión acumulada en millones de dólares americanos. Diciembre 2017.

\begin{tabular}{lcc}
\hline \multicolumn{1}{c}{ Proyecto } & & \\
& & \\
& Inversión acumulada a diciembre 2017 & \\
\hline Corredor Logístico & & $23.71 \%$ \\
Puerto Cortés terminal contenedores & 123.39 & $18.34 \%$ \\
Aeropuertos de Honduras & 95.41 & $13.69 \%$ \\
Puerto Cortés terminal gráneles & 71.23 & $11.42 \%$ \\
Recuperación de pérdidas ENEE & 59.40 & $7.80 \%$ \\
Corredor Turístico & 40.58 & $7.52 \%$ \\
Centro Cívico Gubernamental & 39.15 & $7.46 \%$ \\
Carretera a Gracias & 38.80 & $5.71 \%$ \\
Siglo XXI & 29.70 & $3.01 \%$ \\
Aeropuerto de Palmerola & 15.66 & $0.90 \%$ \\
La Lima & 4.69 & $0.45 \%$ \\
Total & 2.33 & $100.00 \%$ \\
\hline
\end{tabular}

Fuente: Elaboración propia con base a información del Informe Anual de Actividades 2017 de la Superintendencia de Alianza Público Privada

En lo que respecta a las condiciones generales del contrato del proyecto de la Terminal Especializada de Contenedores, se pueden mencionar las siguientes : (a) Plazo Contrato de Fideicomiso:30 años, (b) Monto Estimado de Inversión: USD \$ 624 millones, (c) Modalidad de Participación: DFBOT, (d) Operador: Operadora Portuaria Centroamericana (OPC), (e)Fiduciario: Banco Financiera Comercial Honduras (FICOHSA), (f) Fecha de toma de posesión: 04 de diciembre del 2012, (g) Fecha de finalización de la concesión: 30 de agosto 2042. 
Por otro lado, en lo que respecta al proyecto Terminal de Gráneles Sólidos, se indica que : (a) Banco Fiduciario: Banco Atlántida, (b) Plazo Contrato de Fideicomiso: 30 Años, (c) Monto Estimado de Inversión: USD \$ 50 Millones, (d) Modalidad de Participación: DFBOT.

\subsection{Terminal Especializada de Contenedores}

Producto de la inversión mencionada en la tabla 2 y, según indica el informe de la SAPP para el año 2017, la terminal de contenedores a través del servicio prestado a las naves mejoró en gran manera la productividad del puerto. A su vez, se analiza la cantidad de carga que se recibe por importaciones, así como aquella que se envía producto de las exportaciones. En la tabla 3 se aprecia la cantidad de contenedores que se reciben en la terminal de contenedores, producto de la importación y exportación de mercancía. A su vez, se presenta el porcentaje de crecimiento con relación al año inmediato anterior.

Tabla 3 Puerto Cortés. Terminal de Contenedores. Cantidad de Contenedores Importaciones vs. Exportaciones. Diciembre 2018

\begin{tabular}{ccccc}
\hline & \multicolumn{4}{c}{ Cantidad de Contenedores } \\
Año & Crecimiento & Exportación & $\begin{array}{c}\% \\
\text { Crecimiento }\end{array}$ \\
\cline { 2 - 5 } & 152,123 & & 157,219 & \\
\hline 2011 & 157,219 & $3.35 \%$ & 157,088 & $-0.08 \%$ \\
\hline 2012 & 152,903 & $-2.75 \%$ & 155,571 & $-0.97 \%$ \\
\hline 2013 & 153,017 & $0.07 \%$ & 156,522 & $0.61 \%$ \\
\hline 2014 & 166,705 & $8.95 \%$ & 168,119 & $7.41 \%$ \\
\hline 2015 & 166,136 & $-0.34 \%$ & 168,109 & $-0.01 \%$ \\
\hline 2017 & 175,658 & $5.73 \%$ & 173,902 & $3.45 \%$ \\
\hline 2018 & 175,025 & $-0.36 \%$ & 179,606 & $3.28 \%$ \\
\hline
\end{tabular}

Fuente: Elaboración propia con base al Informe Anual de Actividades 2018 de la Superintendencia de Alianza Público Privada y el Informe Anual Estadístico de la Empresa Nacional Portuaria para los años 2010-2011 y 2012-2013

En la tabla 4 se detallan los ingresos anuales en millones de dólares que recibe el socio privado, en este caso OPC y el Estado de Honduras por pago de Canon. Así mismo, las sumatorias para los años 2016 y 2017 en lo que respecta a los ingresos de la parte privada, son inconsistentes con los presentados por la SAPP, es decir el informe reporta ingresos inferiores de hasta USD \$ 14 millones para 2016 y USD \$ 2 millones para el año 2017. 
Tabla 4: Ingresos en Millones de dólares americanos. Parte Pública v Privada. Del 2014 al 2018.

Años

\begin{tabular}{lcccc}
\multicolumn{1}{c}{ Descripción } & 2014 & 2015 & 2016 & 2017 \\
\cline { 2 - 5 } & & 86.56 & $81.43^{*}$ & $92.25^{*}$ \\
\hline $\begin{array}{l}\text { Ingresos de la parte } \\
\begin{array}{l}\text { Privada } \\
\text { Canon al Estado de } \\
\text { Honduras }\end{array}\end{array}$ & 71.33 & 14.00 & & 99.03 \\
\hline & 13.43 & & 14.63 & 14.31
\end{tabular}

\begin{tabular}{lllll}
\hline Porcentaje del Canon & $18.83 \%$ & $16.18 \%$ & $17.96 \%{ }^{*}$ & $15.51 \%{ }^{*}$
\end{tabular}

Fuente: Elaboración propia con base al Informe Anual de Actividades 2018 de la Superintendencia de Alianza Público Privada

* Los valores correspondientes a los totales de ingresos que corresponden a la parte privada del Informe Anual de Actividades 2018 de la SAPP presentan inconsistencias en los años 2016 y 2017 con respecto a la sumatoria de los valores mensuales.

En seguimiento a la información publicada por la Superintendencia de Alianzas Público Privadas en el informe 2018 y que se muestra en la figura 1, donde se grafican los incrementos / decrementos de manera porcentual en lo que respecta a los ingresos de los años 2014-2018 conforme al modelo contractual DFBOT suscrito. En la figura 1 y 2 se muestran los incrementos porcentuales en lo referente al ingreso económico para la parte privada como para el Canon del Estado. En la figura 1 se muestran los datos tal y como se encuentran en el informe de la SAPP para el año 2018, sin embargo, se indica que existen inconsistencias en los ingresos que corresponden para la parte privada en los años 2016 y 2017. En la figura 2, se muestran los datos con la corrección correspondiente en lo que respecta a los ingresos de la parte privada para los años 2016 y 2017 respectivamente.

Figura 1: Incremento Porcentual con respecto al año anterior del Ingreso Económico.

Parte Privada vs. Parte Pública. 2015-2018

"Datos 2016 y 2017 con inconsistencias"

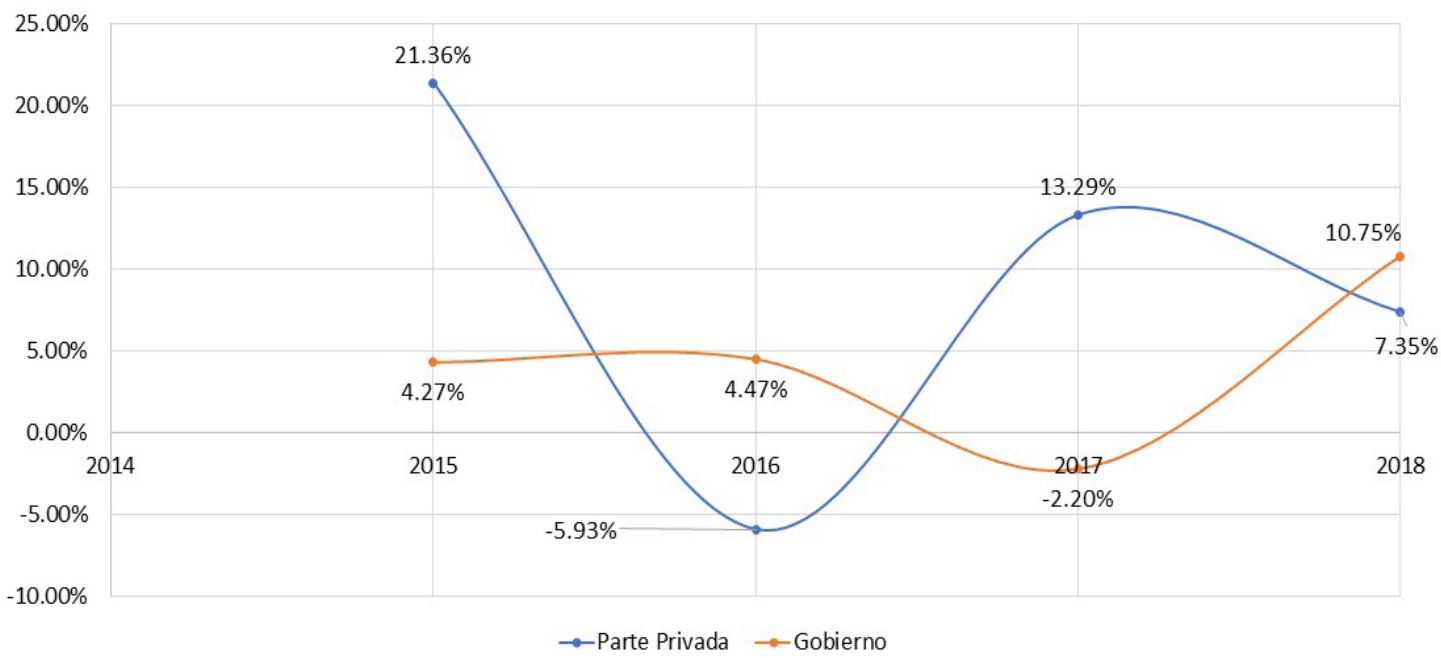

Fuente: Elaboración propia con base al Informe Anual de Actividades 2018 de la Superintendencia de Alianza Público Privada

* Los valores correspondientes a los totales de ingresos que corresponden a la parte privada del Informe Anual de Actividades 2018 de la SAPP presentan inconsistencias en los años 2016 y 2017 con respecto a la sumatoria de los valores mensuales. 
Figura 2: Incremento Porcentual con respecto al año anterior del Ingreso Económico.

Parte Privada vs. Parte Pública. 2015-2018 "Datos 2016 y 2017 Corregidos"

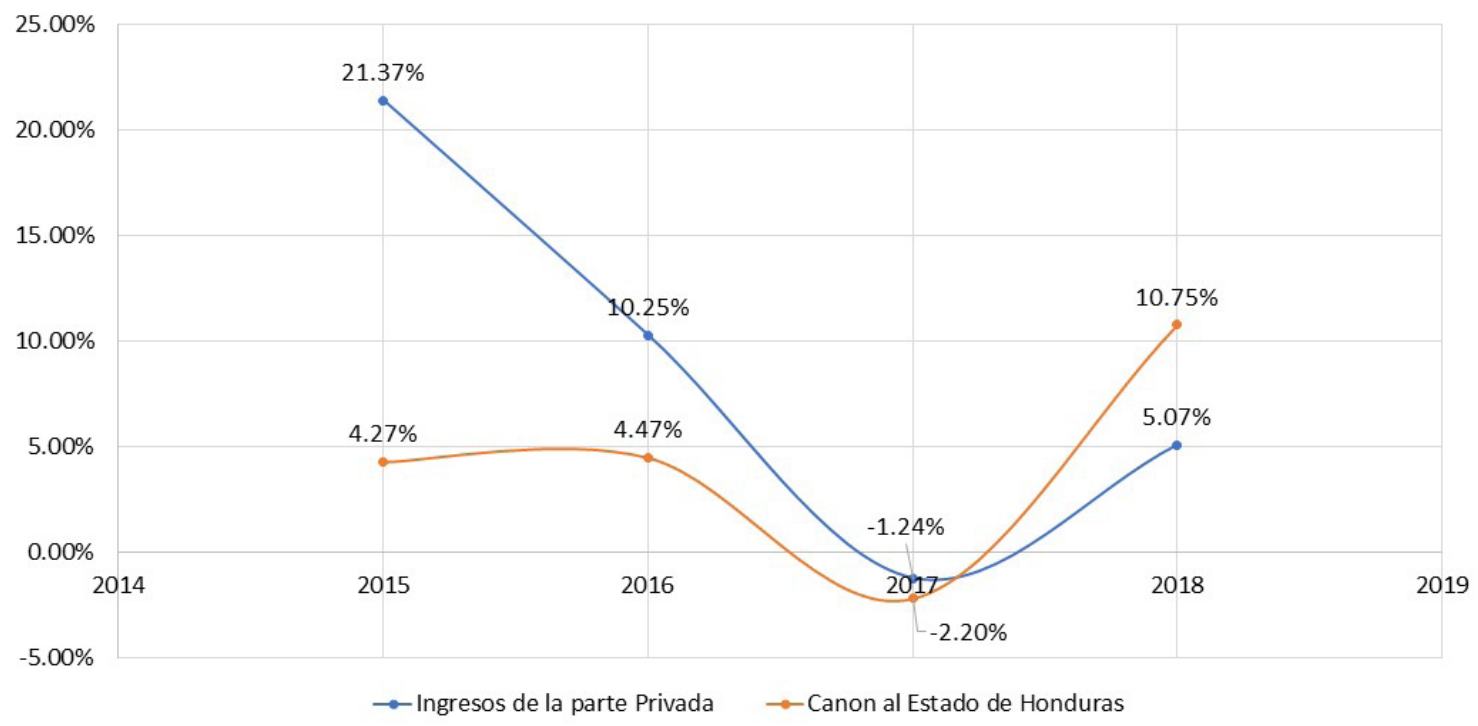

Fuente: Elaboración propia con base al Informe Anual de Actividades 2018 de la Superintendencia de Alianza Público Privada

${ }^{*}$ Los valores correspondientes a los totales de ingresos que corresponden a la parte privada del Informe Anual de Actividades 2018 fueron ajustados para efectos de este análisis.

Del 2015 al 2018 la OPC ha recibido, por manejar Puerto Cortes, en ingresos la cantidad de USD\$446.60 millones de dólares americanos.

\subsection{Terminal de Gráneles Sólidos}

En lo que respecta a la terminal de gráneles solidos no existe información, en el Informe de la SAPP para el año 2018, referente a los ingresos de la parte privada y del Estado, así mismo no se cuenta con información de indicadores de servicio y productividad.

\subsection{ACTIVIDADES RSE}

OPC comenzó sus operaciones en Puerto Cortés en febrero del 2014, bajo el objetivo de la estandarización de procesos, inversiones estratégicas en equipamiento, instalaciones, tecnología y desarrollo del capital humano, todo lo anterior, basado en la cultura del trabajo seguro, siendo estos los pilares de la transformación de Puerto Cortes. En su momento se enfrentaron los siguientes retos: (a) la falta de concientización ambiental, (b) carencia de controles en la utilización de recursos y tratamiento adecuado de los desechos sólidos y líquidos, (c) consumo excesivo de recursos.

Para subsanar los retos mencionados la OPC establece un programa inclusivo de (1) Concientización: (a) charlas de 5 minutos, (b) inducción general desde primer ingreso en el tema ambiental, (c) manual de contratistas haciendo inclusión a este punto ambiental, (d) campaña anual sobre el ambiente y brochure. (2) Controles Operacionales: (a) utilización de las 3R (reducir, reutilizar y reciclar), (b) barrera de contención para el canal de aguas lluvias y se realiza recolección semanal de desperdicios de la ciudad y OPC, (c) contención de derrames, (d) reducción de emisiones. Así mismo, el Programa de Responsabilidad Social Empresarial, el cual está enmarcado dentro de la política de Inversión Social. Este programa se basa en tres pilares fundamentales: (a) educación, (b) medioambiente y (c) salud. 
Tabla 5: Inversión en RSE por parte de OPC y otros actores

\begin{tabular}{|c|c|c|c|}
\hline Pilar & Lugar & Descripción & $\begin{array}{l}\text { Inversión } \\
\text { Millones de USD\$ }\end{array}$ \\
\hline Salud & Hospital Puerto Cortés & $\begin{array}{l}\text { Con el apoyo de diferentes empresas y la comunidad se remodelo las } \\
\text { salas de pediatría, postparto, Puerperio Quirúrgico y Labor y Parto. }\end{array}$ & 0.2950 \\
\hline Medio Ambiente & $\begin{array}{l}\text { Parque Ecológico } \\
\text { OPCito }\end{array}$ & $\begin{array}{l}\text { Nace con el objetivo de reutilizar los materiales en desuso de la } \\
\text { terminal portuaria y de este modo aportar a los proyectos nacionales } \\
\text { de prevención de la delincuencia para las generaciones futuras, } \\
\text { brindándoles un espacio de sano esparcimiento. }\end{array}$ & 0.0097 \\
\hline Educación & Escuela Municipal Emanuel & $\begin{array}{l}\text { Con el fin de apoyar la educación y la inclusión de niños con } \\
\text { discapacidad ha decidido construir la Escuela Municipal Emanuel ya } \\
\text { que actualmente funciona en una casa de habitación la cual cuenta } \\
\text { con barreras arquitectónicas para que los niños puedan recibir sus } \\
\text { clases de la manera más adecuada. }\end{array}$ & $\begin{array}{l}0.1000 \\
\text { (Aún no se ha ejecutado) }\end{array}$ \\
\hline
\end{tabular}

Fuente: Elaboración propia con base a información de Presentación OPC

La inversión reportada por la OPC en conjunto con el apoyo de diferentes empresas en actividades de RSE desde el año 2014 asciende a USD \$ 0.3047 millones de dólares americanos.

En lo que respecta a la reducción de los desechos sólidos en las instalaciones de Puerto Cortés, se han realizado varias actividades con un impacto significativo de reducción entre los años 2016 y 2017 de un 53.59\%. En la figura 3 se presenta las tendencias de Reducción de Desechos Sólidos.

Figura 3: Reducción de Desechos Sólidos en Instalaciones de Puerto Cortés

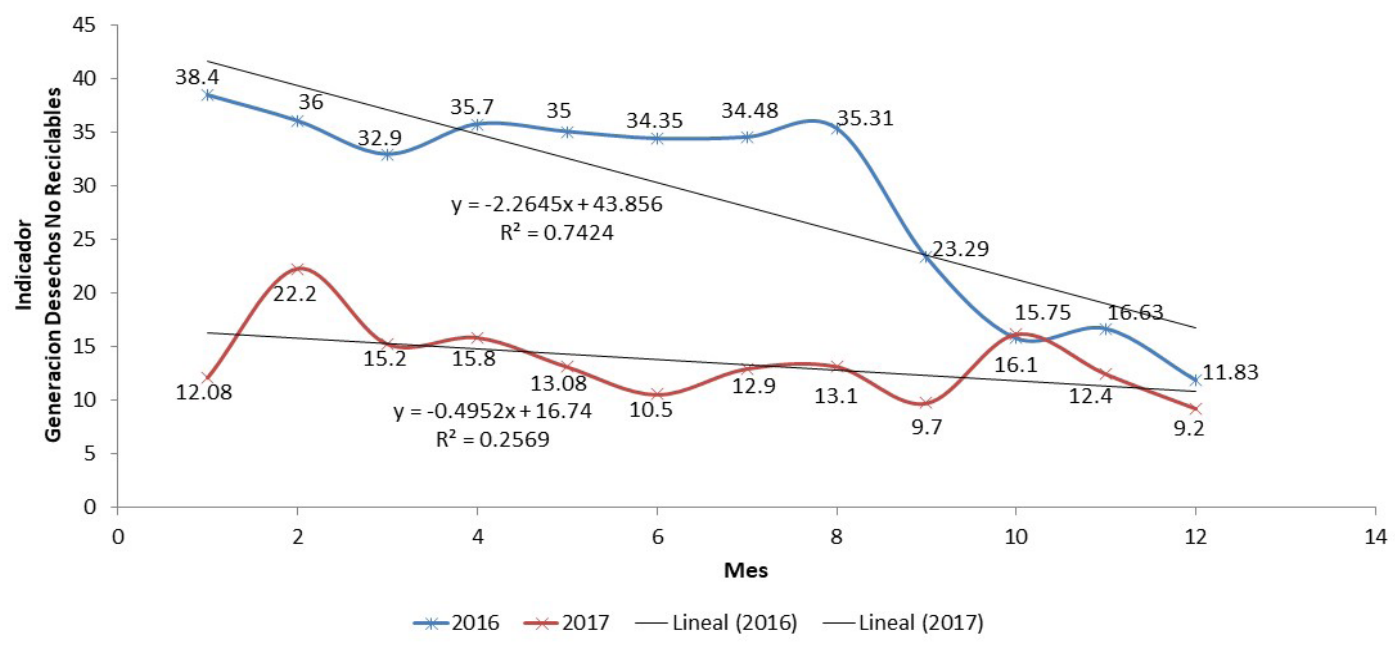

Fuente: Elaboración propia con base a información de la OPC

\section{ANÁLISIS}

En lo que compete a la inversión en el país, a través de la modalidad APP, existen 11 proyectos justificados como APP, se evidencia en la Tabla 2 que el 29.75\% del total de la inversión acumulada al 31 de diciembre del 2017, está alocado para inversión en puertos, específicamente en Puerto Cortés con dos modalidades APP a 
través de DFBOT, una por USD \$ 95.41 millones y la otra por USD \$ 59.40, con un impacto acumulado en dólares americanos de USD \$ 154.81 millones. Una desviación estándar de USD \$ 37.60 millones de dólares y un error estándar de la media por USD \$ 11.30 millones. La inversión en este rubro, supera la media de la inversión del País a través de APP en USD\$ 125.16 millones que equivale a un $422.12 \%$ arriba del promedio. Del análisis y estudio que se muestra en la Tabla 6, se pueden observar los resultados de los estadísticos descriptivos en lo que respecta al Proyecto de la Terminal Especializada de Contenedores. Así mismo, según información obtenida de los informes de la SAPP, para el año 2018 en lo que respecta al proyecto de Puerto Cortes, Terminal de Contenedores, la inversión total que se tiene planificada y programada según lo convenido contractualmente es por USD \$ 624.37 millones, sin embargo, el Informe de la SAPP al 31 de diciembre 2018 indica que solamente se ha cumplido en lo que respecta a inversión, en un $23.75 \%$ de lo pactado.

En lo que respecta a la productividad de la Terminal de Contenedores los informes tanto de la SAPP como de la ENP reportan que la productividad ha mejorado, sin embargo, para efectos del análisis no se tuvo acceso a información de años anteriores, por lo tanto, al momento no hay información que corrobore que efectivamente existe una mejora en la productividad de este. Al analizar el resultado de los estadísticos descriptivos mostrados en la tabla 6, en lo que respecta a los tiempos de mejora en la atención, se observa que la media de atención se encuentra en 1.27 minutos, con una desviación estándar de 0.47 minutos y un tiempo máximo de atención de 2 minutos; sin embargo, el parámetro de medición utilizado para este indicador es de 20 minutos, valor que se encuentra fuera del rango del análisis estadístico. Así mismo, en lo que respecta al tiempo de inicio, descarga el 90\% de operación en minutos, la media de atención oscila en los 12.73 minutos, con una desviación estándar de 6.37 minutos y un tiempo máximo de atención de 21 minutos, pero el parámetro de medición utilizado para este indicador es de 30 minutos.

Tabla 6: Estadística Descriptiva

\begin{tabular}{|c|c|c|c|c|c|c|c|}
\hline $\begin{array}{l}\text { Estadística } \\
\text { Descriptiva }\end{array}$ & $\begin{array}{l}\text { Inversión } \\
\text { Acumulada } \\
\text { Millones de } \\
\text { USD\$ }\end{array}$ & $\begin{array}{l}\text { Tiempo inicio } \\
\text { descarga } \\
\text { promedio } \\
\text { minutos }\end{array}$ & $\begin{array}{l}\text { Tiempo inicio } \\
\text { descarga 90\% } \\
\text { operaciones } \\
\text { minutos }\end{array}$ & $\begin{array}{l}\text { Cantidad de } \\
\text { Contenedores } \\
\text { Importación } \\
\text { Unidades }\end{array}$ & $\begin{array}{l}\text { Cantidad de } \\
\text { Contenedores } \\
\text { Exportación } \\
\text { Unidades }\end{array}$ & $\begin{array}{l}\text { Ingreso parte } \\
\text { privada } \\
\text { Millones en } \\
\text { USD\$\$ }\end{array}$ & $\begin{array}{l}\text { Canon del } \\
\text { Gobierno } \\
\text { Millones en USD\$ }\end{array}$ \\
\hline Rango & 121.10 & 1.00 & 19.00 & $23,535.00$ & $24,035.00$ & 27.70 & 2.42 \\
\hline Valor menor & 2.30 & 1.00 & 2.00 & $152,123.00$ & $155,571.00$ & 71.33 & 13.43 \\
\hline$Q 1=25$ & 15.70 & 1.00 & 8.00 & $152,932.00$ & $156,664.00$ & 76.38 & 13.72 \\
\hline$Q 2=50$ & 39.10 & 1.00 & 10.00 & $161,678.00$ & $162,664.00$ & 86.56 & 14.31 \\
\hline$Q 3=75$ & 71.20 & 2.00 & 20.00 & $172,945.00$ & $172,456.00$ & 95.64 & 15.24 \\
\hline Valor mayor & 123.40 & 2.00 & 21.00 & $175,658.00$ & $179,606.00$ & 99.03 & 15.85 \\
\hline RIC: Q3-Q1 & 55.50 & 1.00 & 12.00 & $20,013.00$ & $15,792.00$ & 22.65 & 2.13 \\
\hline Límite inferior: Q1-1.5 (RIC) & -67.55 & -0.50 & -10.00 & $122,912.50$ & $132,976.00$ & 42.41 & 10.52 \\
\hline Límite superior: Q3+1.5 (RIC) & 154.45 & 3.50 & 38.00 & $202,964.50$ & $196,144.00$ & 129.62 & 18.43 \\
\hline Media & 29.65 & 1.27 & 12.73 & $162,348.00$ & $164,517.00$ & 86.12 & 14.44 \\
\hline Mediana & 39.10 & 1.00 & 10.00 & $161,678.00$ & $162,664.00$ & 86.56 & 14.31 \\
\hline Moda & & 1.00 & 10.00 & & & & \\
\hline Error estándar de la media & 11.30 & 0.14 & 1.92 & $3,480.00$ & $3,257.00$ & 4.72 & 0.40 \\
\hline Desviación estándar & 37.60 & 0.47 & 6.37 & $9,844.00$ & $9,212.00$ & 10.55 & 0.90 \\
\hline Correlación Pearson & & & & & & & 0.821 \\
\hline \multicolumn{8}{|l|}{ Covarianza } \\
\hline $\mathrm{N}$ & 11 & 11 & 11 & 8 & 8 & 5 & 5 \\
\hline
\end{tabular}

Fuente: Elaboración propia con información obtenida a través de Minitab 19 de información de Informes SAPP y ENP. 
En lo que respecta a la cantidad de contenedores que están transitando por la terminal, tanto para importación como exportación, en la tabla 3 se muestra el movimiento de contenedores de los años 2011 al 2018. Al analizar la cantidad de contenedores que han transitado por la terminal, en lo que respecta a la importación, se ha tenido un crecimiento de un $15.02 \%$ si se compara los resultados del año 2018 con respecto al 2011. Sin embargo, hay que tomar en consideración que la concesionaria OPC entró en operaciones de la terminal en diciembre 2012, por lo tanto, si se quiere tener un porcentaje real de crecimiento desde que la OPC tomó posesión, el crecimiento es de un $14.46 \%$.

Los resultados de las estadísticas descriptivas, la cantidad media de contenedores que pasan por la terminal para importación es de 162,348, con una desviación estándar de 9,844 contenedores y un error estándar de la media por 3,480. Para efectos de la exportación, el crecimiento ha sido de un $14.24 \%$ en lo que respecta a la comparación del año 2018 al 2011, sin embargo, el crecimiento en relación al año 2013 es de un 15.45\%. La cantidad media de contenedores para la exportación es de 164,517, con una desviación estándar de 9,212 contenedores y un error estándar de la media de 3,257.

En relación a los ingresos, tanto para la parte privada (USD \$ 430.59 Millones) como para el gobierno (USD\$ 72.21) para los años 2014-2018, se detectó inconsistencias en lo que respecta a los ingresos de la parte privada para los años 2016 y 2017 respectivamente. Los ingresos reportados para el 2016 tienen USD\$14 millones de menos y para el 2017, USD $\$ 2$ millones de menos. Por lo tanto, los ingresos reales de la parte privada ascienden a USD \$ 446.60 millones de dólares. Según la revisión realizada, lo que respecta al Canon del Estado no se evidenciaron diferencias.

Al analizar los estadísticos descriptivos, se observa que la media de ingresos de la parte privada por año es de USD \$ 86.12 millones, con un valor máximo de USD\$99.03 millones por año y una desviación estándar de USD\$ 10.55 millones. Por el lado del Canon del Estado, del 2014 al 2018 se han recibido USD\$ 72.21 millones, con una media por año de USD $\$ 14.44$ millones, una desviación estándar de 0.90 millones; Un valor mínimo de USD \$ 13.43 millones y un máximo de USD \$15.85 millones.

La relación de los ingresos financieros tanto para la parte privada como para el Estado de Honduras, según lo pactado en el modelo DFBOT, el índice de la relación lineal es de 0.821, es decir existe una correlación positiva entre los ingresos para ambas partes. Por otro lado, la dependencia es directa entre las variables de análisis.

En la figura 3, se muestra el gráfico de correlación de Pearson con relación a los Ingresos tanto para la parte privada como para el Canon del Estado de Honduras, así mismo, el indicador de correlación es de 0.821.

Método

Tipo de correlación Pearson

Filas utilizadas $\quad 5$

Correlaciones

C1

$\overline{\mathrm{C} 2} \quad 0.821$

En lo que respecta a la covarianza, el indicador es el siguiente:

Covarianzas

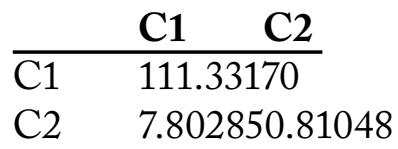


Figura 4: Gráfica para la Correlación de Pearson. Ingresos de la parte privada vs al Canon del Estado de Honduras

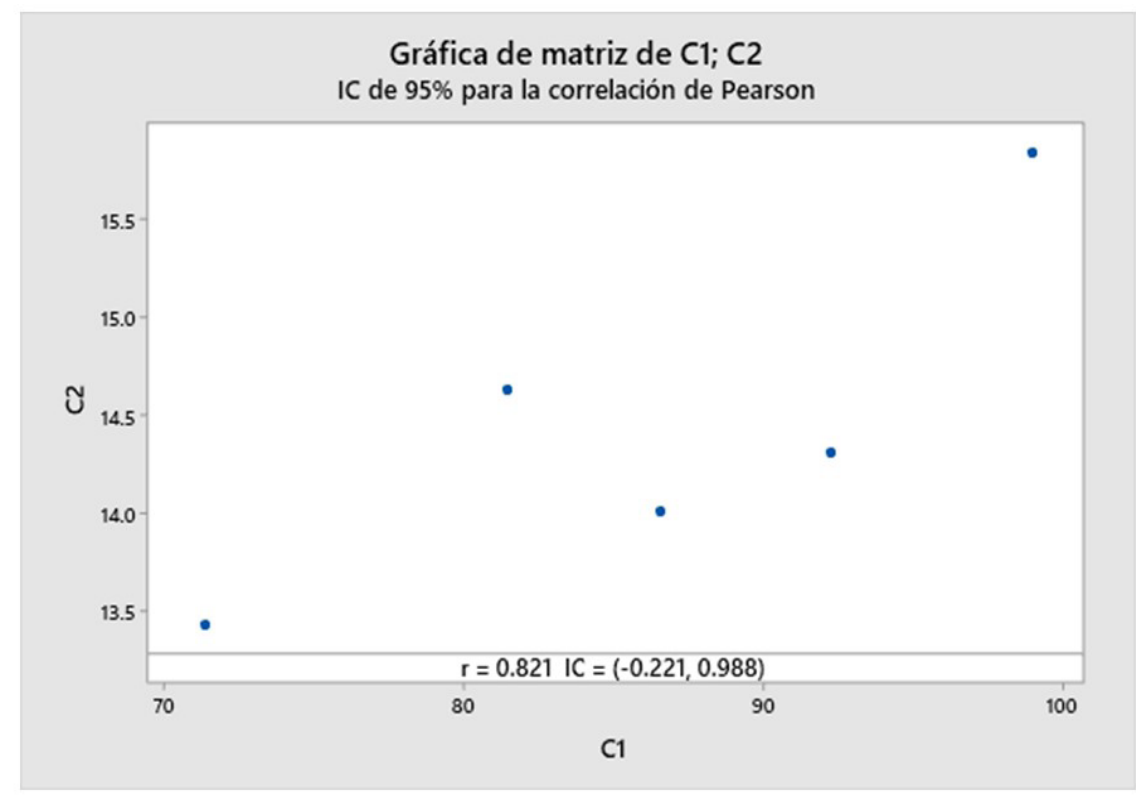

Fuente: Elaboración propia a través de Minitab 19con base a información de Informes SAPP y ENP.

Por lo tanto, F = f(RseApp) donde RseApp = RseApp1, RseApp2, RseApp3...

\section{App (Parte Privada):}

\section{Tabla 7: Análisis Parte Privada}

\begin{tabular}{lr}
\hline Descripción & $\begin{array}{r}\text { Monto en Millones de } \\
\text { dólares americanos }\end{array}$ \\
\hline Inversión por $\mathbf{3 0}$ años & 624.00 \\
Amortización lineal por año & 20.80 \\
Inversión real al 2018 & 148.29 \\
Ingresos medio por año & 86.12 \\
Ingresos proyectados (simple) a 30 años & $2,583.60$ \\
Inversión RSE 2014-2018 & 0.30 \\
\hline
\end{tabular}

Fuente: Elaboración propia con base a información generada en los Informes SAPP, COALIANZA y ENP.

La media de ingresos por año ha sido de USD\$ 86.12 millones y solamente se han invertido al 2018 la cantidad de USD \$ 148.28 millones, por lo tanto, haciendo una matemática simple y depreciando la inversión a 30 años según las condiciones DFBOT, la inversión para el socio privado es bastante rentable, ya que según el contrato se justificó la transferencia del riesgo a la parte privada. 


\section{App (Parte del Estado):}

\section{Tabla 8: Análisis del Estado de Honduras}

\begin{tabular}{lr} 
Descripción & Monto en Millones de dólares americanos \\
\hline Inversión a 30 años & 0.00 \\
\hline Ingreso medio por año & 14.44 \\
\hline Ingresos proyectados (simple) a 30 años & 433.20
\end{tabular}

Fuente: Elaboración propia con base a información generada en los Informes SAPP, COALIANZA y ENP.

Por los resultados obtenidos previamente, se observa a simple vista que, desde el punto de vista de negocio para la parte privada, es un negocio bastante rentable. Al igual, el Estado de Honduras también reporta ingresos con el hecho de concesionar la terminal especializada de contenedores.

Es necesario realizar análisis posteriores con información adicional y veraz para tener una valoración más completa de los resultados financieros para el caso de las Alianzas Público Privadas en puertos hondureños y las actividades RSE.

Así mismo, se deberán realizar evaluaciones posteriores, en el sentido de valorar qué le conviene más al Estado de Honduras, si administrar directamente los puertos o mantener la dinámica de APP para el resto de los puertos hondureños.

El análisis de inversión de la Responsabilidad Social Empresarial es bastante simple, en el sentido que la inversión por este rubro reportada por la OPC asciende a USD $\$ 0.3047$ millones de dólares americanos y los ingresos de la compañía del año 2015 al año 2018 ascienden a USD \$ 446.60 millones de dólares americanos. Por lo tanto, la inversión en RSE por parte de la OPC en Puerto Cortés para tres años es de 0.068\%.

\section{CONCLUSIONES}

Potenciar a Honduras como el país idóneo para el transporte marítimo de carga, contando con la infraestructura portuaria óptima, ofreciendo servicios con los últimos estándares de calidad, tanto a clientes nacionales como internacionales, ha sido una de las razones fundamentales de promover los modelos de Alianzas Público Privadas en el país, específicamente en el rubro portuario. Se ha examinado la modalidad de contratación, los beneficios financieros para las partes al convenirlo, así como las actividades de Responsabilidad Social Empresarial ejecutadas por la parte privada.

La justificación teórica para la evaluación financiera de los proyectos APP, ha sido muy debatida a nivel mundial, en el sentido que los beneficios son evidentes para el socio privado, más no para la parte pública. Para el caso específico de Honduras, las APP`s están en proceso de madurez y las actividades de RSE que se ejecutan no están orientadas al plan país a largo plazo, por lo tanto, se debe evaluar más detalladamente los beneficios para el Estado de Honduras. 
La OPC ha venido ejecutando varias actividades en lo que respecta a la RSE, se puede percibir que existe una buena ciudadanía y un compromiso profesional con la causa, sin embargo, es una RSE de tipo reactiva más que una RSE estratégica, es necesario que el Estado de Honduras en conjunto con la OPC reorienten el valor compartido al invertir en aspectos sociales y que se desarrolle una verdadera relación simbiótica al reforzar la empresa y la comunidad mutuamente. Al momento el porcentaje de inversión en actividades de RSE es de un $0.068 \%$ en tres años, cuando en promedio, empresas de menor envergadura invierten hasta un $1 \%$ de sus presupuestos anualmente.

Por otro lado, no existen indicadores que puedan medir de forma consistente y apropiada el desempeño social en las áreas de educación y salud. Se cuenta con algunos datos estadísticos publicados para los años 2016 y 2017 en lo que respecta a la mejoría de manejo de desechos, mas no para los años subsiguientes. El porcentaje de mejoría de un año versus al otro es de un 53.59\%.

Un hallazgo positivo es que, las nuevas instalaciones han sido construidas con la última tecnología y dentro de la planificación prevista. Los informes presentados indican que la productividad ha mejorado, sin embargo, al analizar los resultados de la productividad, se encuentra que la información proporcionada es escasa y solamente hace referencia a un período fiscal, por otro lado, el indicador de referencia de los tiempos de atención está fuera de los límites normales de las mediciones realizadas.

Para efectos del estudio, solamente se pudo obtener información detallada en lo que respecta a los ingresos y a la productividad del proyecto de Terminal de Contenedores en Puerto Cortés. Para el caso de la información del Proyecto de la Terminal de Gráneles, se requiere utilizar otros mecanismos para accesar la información detallada. 


\section{REFERENCIAS}

Araque Padilla, R. A., \& Montero Simo, M. J. (2008 ). La responsabilidad social de la empresa a debate . Barcelona, España: Icaria.

Hodge , G., \& Greve, C. (2009). PPPs: The passage of time permits a sober reflection. Economic Affairs(29(1)), 33-39. doi:10.1111/j.1468-0270.2009.01864.x

Klijn, E., \& Teisman, G. (2005). Public Private Partnerships as the Management of CoProduction: Strategic and Institutional Obstacles in a Difficult Marriage. In The Challenge of Public-Private Partnerships: Learning from International Experience, eds G. Hodge and C. Greve. Cheltenham: Edward Elgar, 95-116.

Alborta, Guillermo R.; Stevenson, Claudia; Triana, Sergio;. (2011). Asociaciones públicoprivadas para la prestación de servicios : Una visión hacia el futuro. Banco Interamericano de Desarrollo, División de Mercados de Capital e Instituciones Financieras (ICF/ CMF). Washington: Banco Interamericano de Desarrollo.

Arthur Anderson and LSE Enterprise. (2000). Arthur AndeValue for Money Drivers in the Private Finance Initiative. London: UK Treasury Task Force. .

Ascheri, M. (2003-2006). Universidad de Roma. I REVISTA DE HISTORIA MEDIEVAL No 14, pp. 7-20.

Berg, S., Pollitt, M., \& Tsuji, M. (2002). Private Initiatives in Infrastructure: Priorities, Incentives and Performance. Aldershot: Edward Elgar.

Bovaird, T. (2004). Public - Private Partnerships in Western Europe and the U.S. : New Growths from Old Roots . edited by Abby Ghobadian, David Gallear.

Comision de las Comunidades Europeas. (2001). Libro Verde: Fomentar un marco europeo para la responsabilidad social de las empresas. Bruselas: COM (2001) .

Comisión Económica para América Latina y el Caribe (CEPAL). (2016). Horizontes 2030: la igualdad en el centro del desarrollo sostenible. Horizontes 2030, LC/G.2660/Rev.1, 171.

Correa, J. G. (2007). “Evolución histórica de los conceptos de responsabilidad social empresarial y Social”. Correa,

Juan G. (2007). Articulo "Evolución histórica de los conceptos de responsabilidad social empr"Semestre Económico", volumen 10 No 20, Correa, Juan G. (2007). Articulo "Evolución histórica de los conceptos de responsabilidad social emp pág. 89-95.

Cui, C., Liu, Y., Hope, A., \& Wang, J. (12 de March de 2018). Review of studies on the public-private partnerships (PPP) for infrastructure projects. Int. J. of Proj. Manag., pág. 22. doi:doi.org/10.1016/j.ijproman.2018.03.004

Edwards, P., \& Shaoul, J. (2003). Partnerships: For better, for worse? Accounting, Auditing \&amp; Accountability Journal(16(3)), 397-421. doi:10.1108/09513570310482345

Froud, J. (2003). The Private Finance Initiative: Risk, uncertainty and the state. Accounting, Organizations and Society, 567-589. doi:doi:10.1016/S0361-3682(02)00011-9

Ghobadian, A., O’Regan, N., Gallear, D., \& Viney, H. (2004). Public-Private Partnerships: Policy and Experience. Basingstoke: Palgrave Macmillan.

Grimsey, D., \& Lewis, M. K. (2004). Public-Private Partnerships: The Worldwide Revolution in Infrastructure Provision and Project Finance. Cheltenham: Edward Elgar.

Herrera, J., \& Abreu, J. (2008). Cómo gestionar la responsabilidad social en las pymes Colombianas (How to manage corporate social responsibility in small and middle-sized Colombian. Herrera, J \& Abreu, Jose. (2008). Cómo gestionar la responsabilidad social en las pymes Colombianas (How to manage corporate social rInternational Journal of Good Conscience.,

Herrera, J \& Abreu, Jose. (2008). Cómo gestionar la responsabilidad social en las pymes Colombianas (How to manage corporate social responsi 395-425.

Hodge, G. (2004). The risky business of public-private partnerships. . Australian Journal of Public Administration., 37-49. doi:10.1111/j.1467-8500.2004.00400.x

Hodge, G., \& Grave, C. (2007). Public-private partnerships: An international performance review. Public Administration Review. (67(3)), 545-558. doi:10.1111/j.1540-6210.2007.00736.x 
Hodge, G., \& Greve, C. (2010). Public-private partnerships: Governance scheme or language game? Australian Journal of Public Administration(69), S8-S22. doi:10.1111/j.1467-8500.2009.00659.x

Marques, R., \& Berg, S. (2011). Risks, contracts, and private-sector participation in infrastructure. Journal of Construction Engineering and Management(137(11)), 925-932. doi:Marques, R. (2011). Risks, contracts, and private-sector participation in infrastructure. Journal of Construction Engineering and 10.1061/(ASCE)CO.19437862.0000347

McKee, M., Edwards, N., \& Atunc, R. (2006). Public-private partnerships for hospitals. Bulletin of the World Health Organization(84(11)), 890-896. doi:10.2471/BLT.06.030015

NAO. (2003). Managing Resources to Deliver Better Public Services. London.: National Audit Office.

Neto, D. d., Cruz, C. O., Rodrigues, F., \& Silva, P. (2016). Bibliometric Analysis of PPP and PFI Literature: Overview of 25 Years of Research. Journal of Construction Engineering and Management, 142(10). doi:DOI: 10.1061/ (ASCE)CO.1943-7862.0001163.

Nisar, T. M. (2007). "Value for money drivers in public private partnership schemes". International Journal of Public Sector Management, Vol. 20 Iss 2, 147 - 156. doi:Tahir M. Nisar, (2007),"Value for money drivers in public private partnership schemes", International Journal of Public SePermanent link to this document: http://dx.doi. org/10.1108/09513550710731508

Osborne, S. (2001a). Introduction: Understanding Public-Private Partnerships in International Perspective: Globally Convergent or Nationally Divergent Phenomena?' In Public-Private Partnerships: Theory and Practice in International Perspective. ed. S. Osbo.

Osborne, S. (ed. 2001b). Public-Private Partnerships: Theory and Practice in International Perspective. New York: Routledge.

Perrot, J.-Y., \& Chatelus, G. (eds. 2000). Financing of Major Infrastructure and Public Service Projects: Public Private Partnerships, Lessons from French Experience Throughout the World. Paris: Presses de l'ecole nationale des Ponts et Chaussees.

Porter, M. E., \& Kramer, M. R. (2006). Estrategia y sociedad: El vinculo entre ventaja competitiva y responsabilidad social corporativa. Harvard Business Review, Porter M., Kramer M. (10 de octubre de 2009). Estrategia y sociedad. E1 vinculo entre.

Ruiz Malbarez, M. C., \& Romero González, Z. (2011). LA RESPONSABILIDAD SOCIAL EMPRESARIAL Y LA OBSOLESCENCIA PROGRAMADA. SABER, CIENCIA Y Libertad, 127-135.

Sarmiento del Valle, S. (2011). La Responsabilidad Social Empresarial: gestion estrategica para la supervivencia de las empresas. Dimens. empres., 6-15.

Savas, E. (2000). Privatisation and Public-Private Partnerships. New York: Chatham House Publishers and Seven Bridges Press.

Shaoul, J. (2005). A critical financial analysis of the Private Finance Initiative: Selecting a financing method or allocating economic wealth? Critical Perspectives on Accounting(16(4)), 441-471. doi:10.1016/j.cpa.2003.06.001

Tiong, R. (1 de 6 de 1990). Comparative study of BOT projects. Journal of Management in Engineering, 107-122. doi:10.1061/(ASCE)9742-597X(1990)6:1(107)

Vaillancourt Rosenau, P. (ed. 2000). Public-Private Policy Partnerships. Cambridge, MA : The MIT Press.

Van Ham, J., \& Koppenjan, J. (2001). Building Public-Private Partnerships. Assessing and Managing Risks in Port Development. Public Management Review 4(1), vol. 4( no. 1), 593-616.

Van Ham, J., \& Koppenjan, J. (8-10 de April de 2002). Partnerships Passing in the Night? Paper presented at IRSPM 5. Edinburgh.

Villalba-Romero, F., \& Liyanage, C. (2016). "Implications of the use of different payment models : The context of PPP Road Projects in the UK”. International Journal of Managing Projects in Business, 9 Issue: 1, 11-32. doi:10.1108/ IJMPB-09-2015-0095

Wettenhall, R. (2003). “The Rhetoric and Reality of Public-Private Partnerships. ” Public Organization Review 3 (1), 
Springer: 77-107.

Wettenhall, R. (2005). The Public - Private Interface: Surveying the History. The Challenge of Public - Private

Partnerships: Learning from International Experience, edited by Graeme Hodge and Carsten Greve, 22 - 43.

Laboral: Un Análisis Para El Mercado Laboral Colombiano 2001- 2006”,Desarrollo y Sociedad, núm. 65, pp. 191-234.

\section{BIBLIOGRAFÍA}

Perrot, J.-Y., \& Chatelus, G. (eds. 2000). Financing of Major Infrastructure and Public Service Projects: Public Private Partnerships, Lessons from French Experience Throughout the World. Paris: Presses de l'ecole nationale des Ponts et Chaussees.

\section{NOTAS AL FINAL}

I. Recuperado de https://sapp.gob.hn/services/puertos/

II. Recuperado de https://sapp.gob.hn/services/puertos/

III. Recuperado de http://coalianza.gob.hn/es/cartera-de-proyectos/cartera-app-nacional/puertos/proyectoterminal-de-contenedores-y-carga

IV. Recuperado de https://papers.ssrn.com/sol3/papers.cfm?abstract_id=3372915

V. Ficha de trabajo 2, Disertación Doctoral 1-DDE-UNAH-2019, Dr. Javier Torres Vindas.

VI. Recuperado de https://sapp.gob.hn/wp-content/uploads/INFORME-ANUAL-DE-ACTIVIDADES-2017. pdf

VII. Recuperado de https://sapp.gob.hn/wp-content/uploads/INFORME-FINAL-2018.pdf

VIII. Recuperado de http://coalianza.gob.hn/es/cartera-de-proyectos/cartera-app-nacional/puertos/proyectoterminal-de-graneles-solidos

IX. Recuperado de https://sapp.gob.hn/wp-content/uploads/INFORME-FINAL-2018.pdf

X. Recuperado de http://www.enp.hn/estadistico.html

XI. Recuperado de https://sapp.gob.hn/wp-content/uploads/INFORME-FINAL-2018.pdf

XII. Recuperado de https://sapp.gob.hn/wp-content/uploads/INFORME-FINAL-2018.pdf

XIII. Recuperado de https://sapp.gob.hn/wp-content/uploads/INFORME-FINAL-2018.pdf

XIV. http://portalcip.org/wp-content/uploads/2019/06/Premio-Mar\%C3\%ADtimo-OPC-Honduras.pdf

XV. Recuperado de https://sapp.gob.hn/wp-content/uploads/INFORME-FINAL-2018.pdf

\section{ANEXO}

\section{ANEXO 1}

Los siguientes conceptos son extraídos del contrato para el Diseño, Financiamiento, Construcción, Conservación, Operación y Explotación de la Terminal de Contenedores y Carga general de Puerto Cortés, que suscriben la República de Honduras actuando a través de la Comisión para la Promoción de la Alianza Público-Privada (COALIANZA) y Banco Financiera Comercial Hondureña, S.A., por una parte, y operadora de Puerto Cortés, S.A. de C.V. por la otra. 
CONCEPTO

Administrador portuario.

COALIANZA

DFBOT

Empresa nacional portuaria.

\section{SIGNIFICADO}

Es la persona jurídica, pública o privada encargada de la explotación de una infraestructura portuaria y de la prestación delos servicios portuarios, en una o más terminales portuarias, independientemente de la denominación o título bajo el cual se realiza dicha explotación. En tal sentido, cuando en el Pliego de Condiciones se haga referencia a la administraci6n de una terminal portuaria, se entiende la explotación de dicha infraestructura y la prestación de servicios portuarios en ésta.

Banco Interamericano de Desarrollo.

Es la Comisión para la Promoción de la Alianza Público - Privada, ente desconcentrado de la Presidencia de la República de Honduras creado mediante Decreto $\mathrm{N}^{\circ}$ 143-2010, encargado de gestionar y promover de manera exclusiva los proyectos y procesos de las alianzas públicoprivadas y que es Parte del Contrato.

Es el documento vinculante, los Anexos y Apéndices que lo integran, celebrado entre Gobierno, el Fiduciario de una parte y el Operador.

DFBOT (Design, Finance, Build, Operate and Transfer). Es la modalidad de contratación mediante la cual la OPERADOR se obliga a llevar a cabo el Diseño, Financiamiento, Construcción, Conservación, Operación y Explotación de la Terminal, y finalmente la transfiera al Gobierno al término del Contrato.

Es la moneda o el signo monetario de curso legal en los Estados Unidos de América.

Es el organismo de servicio público, con autonomía, patrimonio y personalidad jurídica propios, creada por la Ley Orgánica de la Empresa Nacional Portuaria, emitida mediante Decreto Numero cuarenta (40), emitido con fecha catorce(14) de octubre de mil novecientos sesenta y cinco (1965) que tiene por objeto el desarrollo económico del país proporcionando servicios e instalaciones adecuadas y eficientes en los puertos marítimos, contando para tal efecto entre otras atribuciones, la administración, explotación y utilización de los servicios y las obras e instalaciones portuarias a su cargo, dentro de las cuales se encuentra la Puerto Cortés; así como coordinar el desenvolvimiento de las actividades portuarias nacionales. 


\section{Entidad financiera}

Entidad financiera internacional.

Son aquellas empresas definidas como tales por la Ley del Sistema Financiero contenida en el Decreto $129-2004$ y sus reformas.

Es una entidad financiera de primera categoría aquella que al momento de la presentación de la garantía tenga la calificación más alta de conformidad a las evaluaciones de Moody's, Standard and Poor's o Fitch Ratings.

Es el contrato de Fideicomiso constituido por la Empresa Nacional Portuaria y COALIANZA y el Banco que aplique en su calidad de institución fiduciaria, el cual fue aprobado por Decreto No. 82-2012 de fecha treinta y uno (31) de agosto de dos mil doce (2012) expedido por el Congreso Nacional, que tiene entre otras finalidades: recibir los recursos relativos al Diseño, Financiamiento, Construcción, Conservación, Operación y Explotación de la Terminal y administrarlos para cubrir los costos de operación y mantenimiento y garantizar dichos financiamientos.

Dependiendo del tipo de área de inversión puede ser el

Difeicomiso.

Financiamiento

Fondo de Tasas por Servicios Prestados por COALIANZA.

Infraestructura portuaria. Banco Financiera Comercial Hondureña, S.A. en su calidad de institución fiduciaria, en el Fideicomiso o Banco Atlántida.

Son los recursos que obtenga el OPERADOR provenientes del Endeudamiento Garantizado Permitido contratado con Acreedores Permitidos para ser aplicados única y exclusivamente para el cumplimiento del objeto del Contrato.

Es el fondo constituido con los aportes realizados por el OPERADOR por concepto de tasa por los servicios prestados por COALIANZA, establecido en el artículo veintinueve (29) del Decreto $\mathrm{N}^{\circ} 143-2010$ de la Ley de Promoción de la Alianza Público-Privada.

La tasa será del dos por ciento (2\%) del total de la Inversión Referencial del Proyecto.

Son las obras civiles e instalaciones mecánicas, eléctricas y electrónicas, fijas y flotantes, construidas o ubicadas en la Terminal y a cargo o bajo la responsabilidad del OPERADOR, necesarias para el cumplimiento del objeto del Contrato. 
Es el resultado de deducir el ISV de la totalidad de los ingresos brutos obtenidos por el OPERADOR, directa o indirectamente, derivados de la Explotación de la Terminal. No se considerarán como ingresos los montos que pudiera

Ingresos netos.

ISV

Lempira

Leyes Aplicables

Puerto o Puerto Cortés

Recinto Portuario.

Súper intendencia de alianzas público privadas

Tarifa recibir el OPERADOR por el reembolso del pago de los servicios públicos a los que se refiere la Cláusula 6.33 el Contrato o los ingresos que perciba alguna de sus Empresas Vinculadas en el servicio de practicaje y/o remolcaje con recursos propios.

Es el Impuesto Sobre Ventas a que se refiere el Decreto $\mathrm{N}^{\circ} 24$ Ley del Impuesto Sobre Ventas o normas que lo sustituyan.

Es la moneda de curso legal de la Republica de Honduras.

Es el conjunto de normas o disposiciones legales y reglamentarias hondureñas de carácter general que regulan el Concurso, el Pliego de Condiciones y el Contrato. Este conjunto de normas incluye, de manera enunciativa mas no limitativa, a la Constitución de la Republica de Honduras, las normas con rango de ley y sus reglamentos, acuerdos y resoluciones, que pueda dictar cualquier Autoridad Gubernamental competente, de conformidad con su ley de creación, las que serán de observancia obligatoria para las Partes.

Para efectos del presente Contrato, es la localidad geográfica y unidad económica denominada Puerto Cortés, localizado en la República de Honduras donde se ubican las terminales, infraestructuras e instalaciones, terrestres y acuáticas, naturales o artificiales, acondicionados para el desarrollo de actividades logísticas y portuarias.

Espacio comprendido entre las obras de abrigo o línea externa de demarcación del área operativa acuática y el limite perimetral terrestre del área en que se ubican las instalaciones portuarias.

Ente encargado de la regulación, control y seguimiento de la realización de obras y prestación de servicios mediante Alianzas Público Privadas.

Es la contraprestación económica que cobrara la OPERADOR por la prestación de los Servicios Estándar y los Servicios Especiales de acuerdo a lo previsto en el Contrato, sin incluir los impuestos que resulten aplicables. 


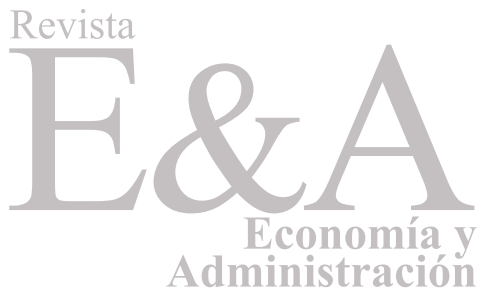

